\title{
CONJUGATE HEAT TRANSFER ANALYSIS OF THE FILM CONDENSATION ON A VERTICAL FIN IMMERSED IN A POROUS MEDIUM
}

\author{
O. Bautistar ${ }^{\mathrm{a}}$, F. Méndez ${ }^{\mathrm{b}}$ and P. Tamayo ${ }^{\mathrm{a}}$ \\ ${ }^{a}$ Sección de Estudios de Posgrado e Investigaciól ESIME-Azcapotzalco, Instituto Politécnico \\ Nacional, 02550 México D. F. Mexico, obautista@ipn.mxr, http://www.ipn.mx \\ ${ }^{b}$ Departamento de Termoenergía y Mejoramiento Ambiental, Universidad Nacional Autónoma de \\ México,04510 México,D.F., Mexico,fmendez@servidor.unam.mx, http://www.unam.mx
}

Keywords: film condensation, porous medium, vertical fin.

\begin{abstract}
In the present work, we treat theoretically the conjugate film-condensation heat transfer process on a vertical fin embedded in a homogeneous porous medium. Due to the finite thermal conductivity of the fin, the simultaneous thermal interaction between the vertical fin and the film condensation is presented. In order to predict the thickness of the condensate, the momentum and energy balance equations of the condensate and the energy equation for the fin are reduced to a nonlinear system of two ordinary differential equations with three non-dimensional parameters: the Jakob number, $J a$, a conjugate heat transfer parameter $\alpha$ and the aspect ratio of the fin $\varepsilon$. Using the limit of $J a \ll 1$ and the boundary layer approximation for the film-condensation process, the nondimensional heat transfer and the overall mass flow rates of condensed fluid have been obtained as functions of the involved nondimensional parameters.
\end{abstract}




\section{INTRODUCTION}

Heat transfer analysis of film condensation in a porous medium is an active area basically related with the thermal design of heat exchangers. Here, we are interesting to analyze the steady laminar film condensation over a vertical fin taking into account that the simultaneous thermal interaction between both regions is crucial to understand basic aspects of the heat transfer connected with the thermal control and performance of the fin. Since the pioneer paper of Cheng (1981) to study the steady laminar film condensation outside a inclined cone immersed in a porous medium filled with a dry saturated vapor, several corrections and modifications have been explored during the past decades in order to improve the simple Cheng's theory. Under steady state conditions, the state of the art can be found in Nield and Bejan (1998) and Kaviany (1995). However, one important aspect that still has not been considered in the specialized literature corresponds just to the analysis of this conjugate heat transfer problem. From this point of view, the thermal interaction occurs only between the vertical faces of the fin and the adjacent laminar films of the condensate. Because the aspect ratio of the fin defined later is very small compared with the unity, the condensation process on the top of the fin is negligible. The thermal interaction between the fin and the condensate introduces an additional difficulty: the necessity to include non-isothermal conditions for the fin in order to have an adequate description of the involved phenomena. Well-recognized works mostly deal with film condensation over isothermal walls in non-porous ambient. Sparrow and Gregg (1959), among others, solved numerically a set of partial differential governing equations for the gravity driven laminar film condensation on a vertical flat plate. They employed boundary layer theory and similarity methods for a plate at uniform temperature. Other analysis in non-porous media has been documented by Merte (1973) and extended by Koh et al. (1961) and Chen (1961). Although the foregoing works are essential contributions to the study of laminar film condensation, they were only reserved for those situations where the temperature at the surface of the plate has been maintained uniform. However, we notice that this situation is only valid for idealized cases. This was recognized by Patankar and Sparrow (1979) in their numerical study of laminar film condensation on a vertical fin attached to a cooled vertical plate or cylinder. In this work, the condensation process is coupled with the heat conduction within the fin. They used a similarity analysis and concluded that the calculated fin heat transfer is lower than the predicted value obtained by using an isothermal fin model. Wilkins (1980) has shown that an explicit analytical solution is possible for the formulation of Patankar and Sparrow (1979). These contributions reveal that the studies of condensation on extended surfaces form a class by themselves and, for these problems, an estimation of surface area requirements for a condenser using the classical Nusselt analysis is not appropriate. Sarma et al. (1988) studied the condensation process on a vertical fin of variable thickness. By matching the governing equations of the vertical fin and the condensed phase, through appropriate wall condition, they have analyzed the effect of fin geometry on condensation heat transfer and, they found that the influence of this thermal interaction is of primordial importance. For practically the same problem with uniform thickness of the fin, Chen et al. (1994) solved the coupled interaction in the presence of the shear stress at the liquid-vapor interface, pointing out the influence of the dimensionless Prandtl number, $\mathrm{Pr}_{c}$, Jacob number, Ja on the Nusselt number, $\mathrm{Nu}$. Experimental results on film condensation have been correlated by Chen et al. (1987). Later, Méndez and Treviño (1996) solved the problem (using perturbation and numerical techniques) of laminar film condensation on a surface of a thin vertical plate caused by a forced cooling fluid. They showed that the effect of heat conduction through the plate modifies substantially the classical 
Nusselt solution. Similar results were reported in later works (Treviño 1997 and Méndez 1997). Recently, Kibboua and Azzi (2005) developed a numerical analysis to predict the film condensation on an elliptical tube immersed in a porous medium. They consider free and forced convection conditions.

In order to obtain new solutions with non-isothermal conditions, in this paper we analyze the conjugate laminar film condensation on the external sides of a vertical fin embedded in a homogeneous porous medium. Here, we consider the case for which the base of the fin is maintained at a uniform temperature. The heat flux from the condensed phase to the fin is influenced strongly by the presence of the extended surface with finite thermal conductivity because longitudinal and transverse heat conduction effects become significant. In this work, we use perturbation methods and the boundary layer description for the condensed fluid flow to show that the heat conduction effects through the vertical fin depend on three nondimensional parameters: the Jakob number, $J a, \alpha$ and $\varepsilon$. Parameter $\alpha$ represents the competition between the conductance of longitudinal heat conduction in the fin and the conductance for transverse heat convection through the condensate and $\varepsilon$ is the aspect ratio of the fin. We develop an analysis for all values of $\alpha$ and in some cases; we compare the analytical solutions with the results obtained using numerical techniques.

\section{ORDER OF MAGNITUDE ANALYSIS}

The physical model under study is shown in Figure 1. A thin vertical fin of length $L$ and thickness $2 h$ with $2 h \ll L$, is immersed in a homogeneous porous medium filled with a saturated vapor at temperature $T_{s}$. The fin's base is maintained at temperature $T_{0}<T_{s}$, thus generating a heat flux from the saturated vapor present into the porous medium to the body of the fin and creating thin vertical condensed films on both vertical sides of it. We neglect the condensation effects over the top of the fin due to the involved geometric scales and the top of the fin is adiabatic. Furthermore, the existence of a two-phase zone lying between the condensate and vapor regions is not taken into account. It is well known that for gravitydominated flows, the two-phase region is practically absent. Elaborate analysis to consider this zone is widely studied and can be found elsewhere, (Nield 1998 and Kaviany 1995).

Due to the symmetry of the physical model, we consider only for convenience the right hand side of this configuration. Therefore, we select the upper right corner of the fin as the origin of the coordinate system, whose $y$ axis points in the direction normal to the vertical fin and its $x$ axis points down in the longitudinal direction of the fin, i.e., in the direction of the gravity vector. Taking into account that the fin has a finite thermal conductivity, heat conduction effects exist in both longitudinal and transverse coordinates ( $x$ and $y$, respectively) through the extended surface 


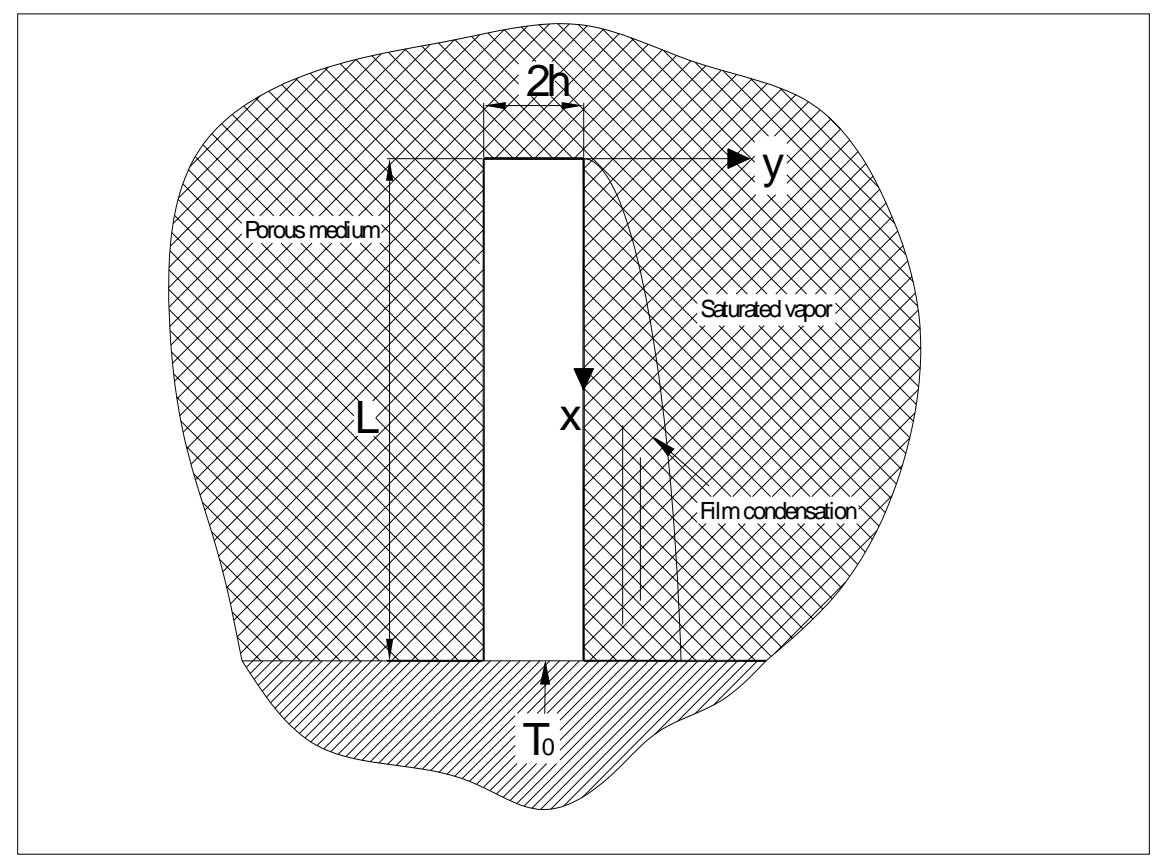

Figure 1: Physical model sketch

An order of magnitude estimate (see for example Bejan 1984) is useful to obtain the nondimensional parameters and the relevant working regimes. Assuming a thin layer of condensing fluid of thickness $\delta_{c}$ (with $\delta_{c} \ll L$ ), the global energy balance can be written as

$$
\frac{\lambda_{m, c} L \Delta T_{c}}{\delta_{c}} \sim \frac{\lambda_{w} h \Delta T_{w L}}{L} \sim h_{f g} \rho_{c} U_{c} \delta_{c} .
$$

Here $\lambda_{w}$ and $\lambda_{m, c}$ are the thermal conductivities of the fin's material and of the porous medium saturated with condensate, respectively. $\Delta T_{c}$ is the characteristic temperature drop at the film condensation and $\Delta T_{w L}$ is the characteristic longitudinal temperature difference along the fin. Both temperatures differences are unknowns and must be determined as a part of this order of magnitude analysis. $h_{f g}$ corresponds to the latent heat of condensation of the saturated vapor, $\rho_{c}$ is the density of the condensed fluid and $U_{c}$ denotes the characteristic longitudinal velocity of the condensate. The order of magnitude of this characteristic velocity can be easily obtained from the Darcy's velocity and in this case $U_{c} \sim K\left(\rho_{c}-\rho_{v}\right) g / \mu_{c}$, where $K$ is the permeability of the porous medium, $g$ is the gravity acceleration, $\mu_{c}$ is the dynamic coefficient of viscosity and $\rho_{c}$ and $\rho_{v}$ are the densities of the condensate and saturated vapor, respectively. For simplicity, we will follow a simple Darcian formulation to describe the hydrodynamics of the condensate. However, the present analysis can be readily extended to include non-Darcian models. Combining the first and third terms of the relationship (1), therefore, we show that a representative global thickness of the condensate layer related to the length of the fin is of order 


$$
\frac{\delta_{c}}{L} \sim\left(\frac{J a}{R a} \frac{\Delta T_{c}}{\Delta T}\right)^{1 / 2} \text {, with } J a=C \frac{c_{c} \Delta T}{h_{f g}} \text { and } R a=\frac{K g L\left(\rho_{c}-\rho_{v}\right)}{\mu_{c} \alpha_{m, c}} .
$$

$J a$ corresponds to the well-known Jakob number defined as the ratio of the heat conducted through the liquid to the latent heat released during the condensation process. $R a$ is the local Rayleigh number, which was first introduced by Cheng (1981) and has been identified as the effect of the equivalent buoyancy-induced fluid motion on film-condensation heat transfer process, (Incropera 1996). $\Delta T=T_{s}-T_{0}$ and $\alpha_{m, c}$ is the effective thermal diffusivity of the porous medium saturated with condensate, defined by $\alpha_{m, c}=\left(\lambda_{m, c} / \rho_{c} c_{c}\right)$ and $c_{c}$ is the specific heat capacity of the condensate. $C$ is a numerical constant adopted to normalize the nondimensional condensate thickness, $C=2$. In general, $J a \ll 1$ and $R a \gg 1$ (Incropera 1996), and thus we can use the boundary layer approximation for the condensed flow in the limit $J a / R a \rightarrow 0$. In the above relationship (2), the ratio $\left(\Delta T_{c} / \Delta T\right)$ is unknown and therefore with the aid of the order of magnitude analysis must be determined. Making a heat flux balance at the vertical side of the fin and obtaining the total temperature drop, $\Delta T$, from the condensed fluid to the base of the fin, we can then write that

$$
\lambda_{m, c} \frac{\Delta T_{c}}{\delta_{c}} \sim \lambda_{w} \frac{\Delta T_{w}}{h} \text { and } \frac{\Delta T_{c}}{\Delta T}+\frac{\Delta T_{w L}}{\Delta T}=1
$$

where $\Delta T_{w}$ is the characteristic transverse temperature difference across the fin. Combining the above relationships together with the first two terms of the relationship (1), with $\delta_{c}$ given by the relationship (2), we obtain that the temperature drop at the condensed fluid, $\Delta T_{c}$ is related to the total temperature drop, $\Delta T$, through

$$
\frac{\Delta T_{c}}{\Delta T}+\frac{1}{\alpha}\left(\frac{\Delta T_{c}}{\Delta T}\right)^{1 / 2}=1
$$

similarly

$$
\frac{\Delta T_{w}}{\Delta T} \sim \frac{\varepsilon^{2}}{\alpha}\left(\frac{\Delta T_{c}}{\Delta T}\right)^{1 / 2} \text { and } \frac{\Delta T_{w L}}{\Delta T} \sim \frac{1}{\alpha}\left(\frac{\Delta T_{c}}{\Delta T}\right)^{1 / 2} .
$$

where the parameters $\alpha$ and $\varepsilon$ are defined by

$$
\alpha=\frac{\lambda_{w}}{\lambda_{m, c}} \frac{h}{L}\left(\frac{J a}{R a}\right)^{1 / 2} \text { and } \varepsilon=\frac{h}{L}
$$

The parameter $\alpha$ represents the competition between the heat conducted by the fin in the longitudinal direction to the heat conducted through the condensate film. Therefore, this parameter defines clearly the conjugate character of the problem. In general, we can distinguish three relevant limits depending on the assumed values of $\alpha$. For $\alpha>1$, the heat conducted through the condensate film has most of the thermal resistance and longitudinal heat flow through the fin has negligible resistance. Thus, no temperature gradients of importance arise in the longitudinal direction. On the other hand, for $\alpha<<1$, resistance to heat flow through condensate film is much smaller than heat conduction in the fin in the longitudinal direction, producing large longitudinal temperature gradients on the plate. We assume that the aspect ratio of the plate, $\varepsilon=h / L$, is very small compared with unity. The 
ratio $\alpha / \varepsilon^{2}$ can assume in general finite arbitrary values. For large values of this parameter compared with unity, we named this limit as the thin thermally fin regime. This case corresponds to the majority of the practical applications. Therefore, from the order relationships (4), (5) and (6), we obtain the following distinguishable limits

$$
\begin{gathered}
\frac{\Delta T_{w L}}{\Delta T} \sim \frac{1}{\alpha}, \frac{\Delta T_{w}}{\Delta T} \sim \frac{\varepsilon^{2}}{\alpha}, \frac{\Delta T_{c}}{\Delta T} \sim 1, \text { for } \alpha \gg 1, \\
\frac{\Delta T_{w L}}{\Delta T} \sim 1, \frac{\Delta T_{w}}{\Delta T} \sim \varepsilon^{2}, \frac{\Delta T_{c}}{\Delta T} \sim 1, \text { for } \alpha \sim 1 \\
\frac{\Delta T_{w L}}{\Delta T} \sim 1, \frac{\Delta T_{w}}{\Delta T} \sim \varepsilon^{2}, \frac{\Delta T_{c}}{\Delta T} \sim \alpha^{2}, \text { for } \alpha \ll 1,
\end{gathered}
$$

which validates the foregoing comments.

\section{MATEMATHICAL FORMULATION}

In this section, we present the nondimensional governing equations needed to solve the conjugate film-condensation problem. Based on the above order of magnitude analysis, we introduce the following non-dimensional variables

Vertical fin:

$$
\theta_{w}(\chi, z)=\frac{T_{s}-T_{w}(x, y)}{T_{s}-T_{0}} ; \chi=\frac{x}{L}, z=\frac{y+h}{h}
$$

Condensed flow:

$$
\theta_{c}\left(\chi, \eta_{c}\right)=\frac{T_{s}-T_{c}(x, y)}{T_{s}-T_{0}}, \Delta(\chi)=\frac{\delta_{c}(x)}{L(J a / R a)^{1 / 2}}, \eta_{c}=\frac{y}{\delta_{c}(x)} ;
$$

In this form, the heat conduction equation for the fin can be written as

$$
\frac{\partial^{2} \theta_{w}}{\partial \chi^{2}}+\frac{1}{\varepsilon^{2}} \frac{\partial^{2} \theta_{w}}{\partial z^{2}}=0
$$

Herein, the variables with the subscript $c$ denote the variables of the condensed phase, those with $w$ denote the variables of the fin. We supposed for simplicity that the top of the fin was adiabatic. Thus, the corresponding boundary conditions in the longitudinal direction at the tip $(\chi=0)$ and the base $(\chi=1)$ are given by

$$
\left.\frac{\partial \theta_{w}}{\partial \chi}\right|_{\chi=0}=\Delta(0)=0 \text { and } \theta_{w}(1, z)=1
$$

and for the transversal direction

$$
\left.\frac{\partial \theta_{w}}{\partial z}\right|_{z=0}=0 \text { and }\left.\frac{\partial \theta_{w}}{\partial z}\right|_{z=1}=\left.\frac{\varepsilon^{2}}{\alpha} \frac{1}{\Delta} \frac{\partial \theta_{c}}{\partial \eta_{c}}\right|_{\eta_{c}=0} .
$$

Introducing the non-dimensional stream function $f_{c}$ defined by 


$$
\begin{array}{r}
\text { Mecánica Computacional } \quad \text { Vol } \quad X X V, \quad p p . \\
u=\frac{\bar{u}}{\left(\alpha_{m, c} R a / L\right)}=\frac{\partial f_{c}}{\partial \eta_{c}} ; \quad v=\frac{L \bar{v}}{\alpha_{m, c}(R a J a)^{1 / 2}}=-\frac{\partial\left(\Delta f_{c}\right)}{\partial \chi}+\eta_{c} \frac{d \Delta}{d \chi} \frac{\partial f_{c}}{\partial \eta_{c}},
\end{array}
$$

where $\bar{u}$ and $\bar{v}$ represent the longitudinal and transverse velocity components in physical units, respectively. The momentum and energy equations for the condensed liquid using the boundary layer approximation, take the form

$$
\begin{gathered}
\frac{\partial f_{c}}{\partial \eta_{c}}=1 \\
\frac{\partial^{2} \theta_{c}}{\partial \eta_{c}^{2}}=J a \Delta^{2}\left\{\frac{\partial \theta_{c}}{\partial \chi}-\frac{\partial f_{c}}{\partial \chi} \frac{\partial \theta_{c}}{\partial \eta_{c}}-\frac{1}{\Delta} \frac{d \Delta}{d \chi} f_{c} \frac{\partial \theta_{c}}{\partial \eta_{c}}\right\} .
\end{gathered}
$$

The boundary conditions associated with the condensed fluid governing equations are

$$
\begin{gathered}
\theta_{c}(\chi, 0)-\theta_{w}(\chi)=f_{c}(\chi, 0)=0 \text { at } \eta_{c}=0 \\
\theta_{c}(\chi, 1)=0 \text { at } \eta_{c}=1 .
\end{gathered}
$$

The normalized non-dimensional thickness of the condensed film $\Delta$, is unknown and must be obtained from the analysis. The energy balance at the condensed-vapor interface gives the evolution of $\Delta$ as

$$
\frac{d \Delta^{2}}{d \chi}=-\left.\frac{\partial \theta_{c}}{\partial \eta_{c}}\right|_{\eta_{c}=1}
$$

The solution of the problem (12)-(20), should provide

$$
\theta_{w}=\theta_{w}(\chi, z: \alpha, \varepsilon, J a) .
$$

In the remainder of this paper we classify the solutions according to the assumed values of $\alpha$, taking advantage of the fact that, in general, $J a$ and $\varepsilon^{2}$ are very small compared with unity. However, for arbitrary values of these parameters, the momentum equation has a trivial solution given as $f_{c}\left(\eta_{c}\right)=1$. Therefore, under small $J a$ and $\varepsilon$, the right hand side of equation (17) can be dropped, and the solution of the energy equation for the condensate liquid yields

$$
\theta_{c}=\theta_{w}(\chi, 0)\left(1-\eta_{c}\right)
$$

A suitable nondimensional heat flux $q^{\prime \prime}$ at the wall or reduced number for this problem $N u_{\chi r}$ is

$$
N u_{\chi r} \equiv \frac{q^{\prime \prime} L}{\lambda_{w, c}\left(T_{s}-T_{0}\right)}\left(\frac{J a}{R a}\right)^{1 / 2}=-\left.\frac{1}{\Delta} \frac{\partial \theta_{c}}{\partial \eta_{c}}\right|_{\eta_{c}=0}=\frac{\theta_{w}(\chi, 1)}{\Delta} .
$$

Thus, the non-dimensional energy balance equation (20) at the interface vapor-condensed fluid transforms to

$$
\frac{d \Delta^{2}}{d \chi}=\theta_{w}(\chi, 1)
$$




\section{THERMALLY THIN FIN REGIME}

The thermally thin wall regime corresponds to the case $\alpha / \varepsilon^{2} \gg 1$. In this regime the temperature variations in the transverse direction in the plate are very small compared with the global temperature difference as predicted by the second of the relationships (7). Therefore, in this regime the temperature of the plate is assumed to depend only in the longitudinal coordinate. The heat conduction equation for the fin can be integrated along the transverse coordinate and after applying the boundary conditions (14) together with the nondimensional condensed fluid temperature profile given by equation (21), we obtain

$$
\alpha \frac{d^{2} \theta_{w}}{d \chi^{2}}=\frac{\theta_{w}}{\Delta} .
$$

In the following subsections, we study the limiting cases of $\alpha>>1$ and $\alpha<<1$, for the thermally thin wall regime.

\subsection{Analysis for the limit $\alpha>>1$}

The system of equations (23)-(24) and the corresponding boundary conditions (13), can be solved by applying a regular perturbation technique, using the inverse of $\alpha$ as the small parameter of expansion. For very large values of the parameter $\alpha$, the non-dimensional temperature of the plate, $\theta_{w}$, changes very little (of order of $\alpha^{-1}$ ) in the longitudinal direction as shown in relationship (7). In order to obtain a solution in this limit, we assume that the non-dimensional temperature of the plate as well as the non-dimensional condensed layer thickness can be expanded in the form

$$
\theta_{w}(\chi)=\theta_{0}(\chi)+\sum_{j=1}^{\infty} \alpha^{-j} \theta_{j}(\chi)
$$

and

$$
\Delta(\chi)=\Delta_{0}(\chi)+\sum_{j=1}^{\infty} \alpha^{-j} \Delta_{j}(\chi)
$$

Introducing these relationships into equations (23) and (24), we obtain after collecting terms of the same power of $\alpha$, the following set of equations

$$
\begin{gathered}
\frac{d^{2} \theta_{0}}{d \chi^{2}}=0, \frac{d \Delta_{0}^{2}}{d \chi}=\theta_{0}, \text { for } \alpha^{0} \\
\frac{d^{2} \theta_{1}}{d \chi^{2}}=\frac{\theta_{0}}{\Delta_{0}}, \frac{2 d\left(\Delta_{0} \Delta_{1}\right)}{d \chi}=\theta_{1}, \text { for } \alpha^{-1} \\
\frac{d^{2} \theta_{2}}{d \chi^{2}}=\frac{\theta_{0}}{\Delta_{0}}\left(\frac{\theta_{1}}{\theta_{0}}-\frac{\Delta_{1}}{\Delta_{0}}\right), \frac{d\left(2 \Delta_{0} \Delta_{2}+\Delta_{1}^{2}\right)}{d \chi}=\theta_{2}, \text { for } \alpha^{-2}
\end{gathered}
$$

etc., with the following initial and boundary conditions

$$
\Delta_{i}(0)=\theta_{0}(1)-1=\left.\frac{d \theta_{i}}{d \chi}\right|_{\chi=0}=0, \text { for all } i
$$


and

$$
\theta_{i}(1)=0, \text { for all } i>0 .
$$

Equations (27) can be integrated with the corresponding initial and boundary conditions (30) giving that $\theta_{0}=1$ and $\Delta_{0}=\chi^{1 / 2}$. Introducing these solutions for $\theta_{0}$ and $\Delta_{0}$ into equations (28) and integrating twice for the energy equation and once for the condensed layer thickness equation, $\Delta_{1}$, we obtain after applying the appropriate initial and boundary conditions

$$
\theta_{1}=\frac{4}{3}\left(\chi^{\frac{3}{2}}-1\right), \Delta_{1}=\frac{2}{3}\left(\frac{2}{5} \chi^{2}-\chi^{\frac{1}{2}}\right)
$$

and in a similar way for the second order terms

$$
\theta_{2}=\frac{8}{45} \chi^{3}-\frac{8}{9} \chi^{3 / 2}+\frac{32}{45}, \Delta_{2}=\frac{2}{15} \chi^{1 / 2}-\frac{1}{75} \chi^{7 / 2}+\frac{8}{45}\left(\chi-\chi^{2}\right)
$$

Therefore, up to the second order, the condensed layer thickness is given by

$$
\Delta=\chi^{1 / 2}\left[1-\frac{2}{3 \alpha}\left(1-\frac{2}{5} \chi^{3 / 2}\right)+\frac{2}{15 \alpha^{2}}\left(1-\frac{1}{10} \chi^{3}+\frac{4}{3}\left(\chi^{1 / 2}-\chi^{3 / 2}\right)\right)\right]+O\left(\alpha^{-3}\right)
$$

and the non-dimensional plate temperature is

$$
\theta_{w}=1-\frac{4}{3 \alpha}\left(1-\chi^{3 / 2}\right)+\frac{32}{45 \alpha^{2}}\left(1-\frac{5}{4} \chi^{3 / 2}+\frac{1}{4} \chi^{3}\right)+O\left(\alpha^{-3}\right) .
$$

The leading term on the right-hand side of the above equations reduces to the classical Cheng (1981) solution for an isothermal plate immersed in a saturated-vapor porous medium.

In order to complete this subsection, we present the method used for the numerical integration of the governing equations. We transform the boundary value problem to an initial value problem by introducing the following non-dimensional variables

$$
\zeta=\frac{\chi}{\alpha^{2 / 3}}, \phi=\frac{\Delta^{2}}{\alpha^{2 / 3}} .
$$

Then the equations transform to the parameter-free form

$$
\frac{d^{2} \theta_{w}}{d \zeta^{2}}=\frac{\theta_{w}}{\phi^{1 / 2}} \text { and } \frac{d \phi}{d \zeta}=\theta_{w}
$$

with $\theta_{w}(0)=\theta_{l}$. The initial conditions are

$$
\frac{d \theta_{w}}{d \zeta}=\theta_{w}-\theta_{l}=\phi=0 \text { at } \zeta=0
$$

for any initial value of $\theta_{l}<1$. The calculations are performed until $\theta_{w}\left(\zeta_{f}\right)=1$ is reached. The value of $\zeta_{f}\left(\theta_{l}\right)$ dictates the appropriate value of $\alpha$ as $\alpha=1 / \zeta_{f}^{3 / 2}$. The asymptotic solution for values of $\zeta \rightarrow 0$, needed to start the numerical integration, takes the form

$$
\theta_{w} \sim \theta_{l}+\frac{4}{3} \theta_{l}^{1 / 2} \zeta^{3 / 2}+\ldots, \phi \sim \theta_{l} \zeta \quad \text { for } \zeta \rightarrow 0 .
$$


As the value of $\alpha$ decreases, $\theta_{l}$ also decreases, reaching the value of $\theta_{l}=0$ for a critical value of $\alpha, \alpha^{*}$, to be obtained as follows. For this critical value of $\alpha$, one can verify that the equations have the following closed form solutions

$$
\theta_{w}=\frac{1}{9} \zeta^{3} \text { and } \phi=\frac{1}{36} \zeta^{4} \text {, for } \alpha=\alpha^{*} .
$$

Here $\zeta_{f}=9^{1 / 3}=2.0801$ and thus, $\alpha^{*}=1 / \zeta_{f}^{3 / 2}=1 / 3$. Therefore, the nondimensional thickness of the condensed layer at the base of the fin gives

$$
\Delta_{f}^{*}=\frac{1}{6 \alpha^{*}}=\frac{1}{2} .
$$

This last result predicts a smaller thickness than the corresponding without porous medium given by Chen (1961).

\subsection{Analysis for the limit $\alpha<\alpha^{*}$}

For small values of $\alpha$, such that $\alpha<\alpha^{*}$ and $\alpha / \varepsilon^{2}>>1$, the thermally thin fin limit is still valid. In this case, a boundary layer develops close to the base of the fin. In order to study the condensation process for very small values of $\alpha$, we introduce the following stretched variables

$$
\xi=\frac{1-\chi}{\alpha^{2 / 3}}, \phi=\frac{\Delta^{2}}{\alpha^{2 / 3}},
$$

transforming the governing equations to

$$
\frac{d^{2} \theta_{w}}{d \xi^{2}}=\frac{\theta_{w}}{\phi^{1 / 2}} \text { and } \frac{d \phi}{d \xi}=-\theta_{w}
$$

with the boundary conditions

$$
\theta_{w}=1 \text { at } \xi=0 \text { and } \theta_{w} \rightarrow 0, \phi \rightarrow 0 \text { for } \xi \rightarrow \infty .
$$

Equations (43) can be written in the phase-space variables as

$$
\phi^{1 / 2}\left[\theta_{w} \frac{d^{2} \theta_{w}}{d \phi^{2}}+\left(\frac{d \theta_{w}}{d \phi}\right)^{2}\right]=1,
$$

with the initial condition $\theta_{w}(0)=0$, which also guarantees the adiabatic condition. The solution can be obtained in closed form as $\theta_{w}=4 \phi^{3 / 4} / \sqrt{6}$, or using the second of the equations (43), we also obtain

$$
\phi=\left[\phi_{f}^{1 / 4}-\frac{1}{\sqrt{6}} \xi\right]^{4} \text { and } \theta_{w}=\frac{4}{\sqrt{6}}\left[\phi_{f}^{1 / 4}-\frac{1}{\sqrt{6}} \xi\right]^{3} .
$$

Here $\phi_{f}$ is the nondimensional value at the base of the fin and is given by $\phi_{f}=(\sqrt{6} / 4)^{4 / 3}=0.52002$. The nondimensional thickness of the condensed layer is then $\Delta_{f}=\alpha^{1 / 3} \phi_{f}^{1 / 2}$, for values of $\alpha \leq \alpha^{*}$. For this case, the condensation layer begins at a very 
well defined position of the fin, $\xi_{\text {wet }}=\sqrt{6} \phi_{f}^{1 / 4}=\sqrt{6}(0.52005)^{1 / 4}=2.0801$. At $\xi>\xi_{\text {wet }}$ $\left(\chi_{\text {wet }}=1-\alpha^{2 / 3} \xi_{\text {wet }}\right.$ ) there is not any condensed fluid at all. The portion of the fin in contact with condensed fluid decreases as the value of $\alpha$ decreases. The fin wets completely for values of $\alpha \geq \alpha^{*}$.

\section{RESULTS}

The analytical and numerical results for the thermally thin fin regime $\left(\alpha / \varepsilon^{2}>>1\right)$ are presented in this section through Figures 2 to 7 . For instance, Figures 2 and 3 show some comparisons between analytical and numerical solutions for the nondimensional thickness of the condensed film, $\Delta$, and for the nondimensional temperature of the fin, $\theta_{w}$, as a function of the nondimensional coordinate $\chi$ and different values of $\alpha$. Specifically, in Figure 2 the differences between both solutions are indistinguishable up to values of $\alpha$ order 2 . In the range $1 / 3<\alpha<2$, we have only plotted the numerical result for $\alpha=0.5$ due to that the perturbation solution stops being valid in this domain. In addition, the corresponding analytical solution for the case of $\alpha=\alpha^{*}=1 / 3\left(\Delta^{*}=\chi^{2} / 2\right)$, is showed and also is indistinguishable of the numerical solution. This last relationship for $\Delta^{*}$ can be readily obtained with the aid of equations (36) and (40) with $\alpha^{*}=1 / 3$. Furthermore, for large values of $\alpha$ both solutions tend to the well-known Cheng (1981) solution $\Delta \sim \chi^{1 / 2}$ (isothermal fin, with $\left.\theta_{w} \sim 1\right)$. In Figure 3, the corresponding analytical and numerical temperature profiles in the vertical fin are also showed as function of the nondimensional coordinate $\chi$ and different values of the conjugate parameter $\alpha$. For decreasing values of $\alpha$, the temperature variations along the longitudinal coordinate are larger, indicating that the temperature of the fin is nearer to the saturated vapor temperature. In this case, the condensation process is limited, as will be showed lines below. Just for the critical value of $\alpha, \alpha^{*}=1 / 3$ the temperature at the top of the fin will be exactly the same as the temperature of the saturated vapor, $\theta_{w}(\chi=0)=0$. Using equations (36) and (40) with this critical value of the parameter $\alpha$ can readily show that $\theta_{w}^{*}\left(\chi, \alpha^{*}\right)=\chi^{3}$. Again, both solutions are indistinguishable.

For values of $\alpha \leq \alpha^{*}$, Figure 4 shows the limiting analytical thickness and temperatures profiles, given by equations (46), as functions of the nondimensional coordinate $\xi$. In this figure, it is clearly showed the dry zone of the fin, which corresponds to values of $\xi \geq 2.08$. On the other hand, in physical units, the mass flow rate of condensed fluid per unit length at the bottom of the fin is then given by

$$
m^{\prime}=\rho_{c} \alpha_{m, c}(R a J a)^{1 / 2} \Delta_{f}(\alpha),
$$

where the numerical and analytical solutions for $\Delta_{f}$ are plotted in Figure 5 in the thermally thin fin approximation. Here, the two-term asymptotic solutions for $\alpha>>1$ and the closed form solution for $\alpha \leq \alpha^{*}$ are also included. The two-term asymptotic solution, gives acceptable results for values of $\alpha \sim 0.6$, while the numerical solution for $\alpha>>1$ offers good results up to values of $\alpha^{*}=1 / 3$. For $\alpha<\alpha^{*}$ the analytical solution given in section 4.2 completes the solution. Finally, If we define the fin efficiency as the ratio of the actual condensate mass flow rate to that obtained by using the isothermal wall $(\alpha \rightarrow \infty)$, then 


$$
\eta=\frac{m^{\prime}}{\left(m^{\prime}\right)_{\infty}}=\Delta_{f},
$$

and by the results of $\Delta_{f}$ obtained in Figure 5 can be readily appreciated that the efficiency of the fin decreases for decreasing values of the conjugate parameter $\alpha$. Finally, in Figure 6 we have plotted the average reduced Nusselt number defined as

$$
N u_{r}=\int_{0}^{1} N u_{\chi r} d \chi=\int_{0}^{1} \frac{\theta_{w}(\chi, 1)}{\Delta} d \chi
$$

as a function of the parameter $\alpha$, using only the numerical results only valid up to the critical value of $\alpha^{*}=1 / 3$. Combining the results of Figures 5 and 6 , we obtain obviously that for decreasing values of the parameter $\alpha$ the nondimensional heat transfer is increased due to the reduction of the thickness of the condensate.

\section{CONCLUSIONS}

In this work, we studied analytically and numerically the conjugate heat transfer filmcondensation process of a saturated vapor on a vertical conducting fin immersed in a porous medium, considering that the base of the fin is found at temperature lower than the temperature of saturated-vapor porous medium. This boundary condition modifies the results obtained in previous works, where the effect of the longitudinal heat conduction through the fin has been neglected. In this particular case, the longitudinal heat conduction must be retained for any value of the parameter $\alpha$. Furthermore, there is a critical value of $\alpha$, $\alpha^{*}=1 / 3$, where the temperature at the top of the fin reaches the temperature of the condensed vapor. For values of $\alpha<\alpha^{*}$, there is a portion of the fin which remains dry. The total mass flow rate of condensed fluid per unit length has been obtained for all values of the involved parametric space and the influence of the parameter $\alpha$ is to reduce the efficiency of the fin for decreasing values of this conjugate parameter. 


\section{FIGURES}

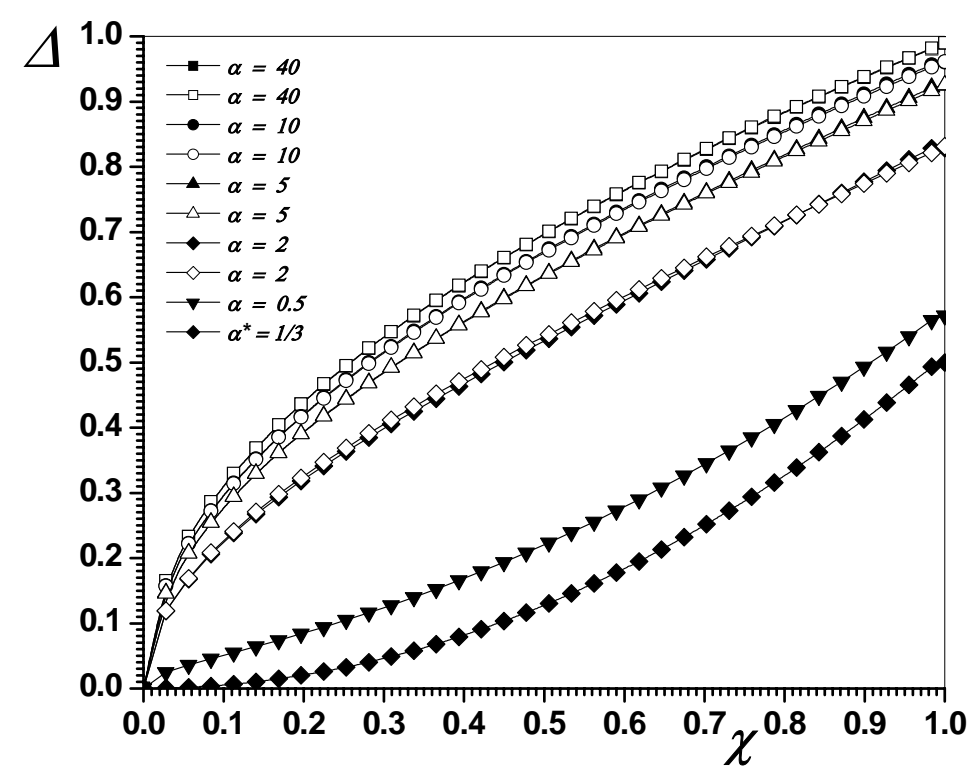

Figure 2: Nondimensional thickness of the condensed film, $\Delta$, as a function of the nondimensional longitudinal coordinate $\chi$, for different values of the conjugate parameter $\alpha$ (black symbols denotes numerical solutions and white symbols are analytical solutions).

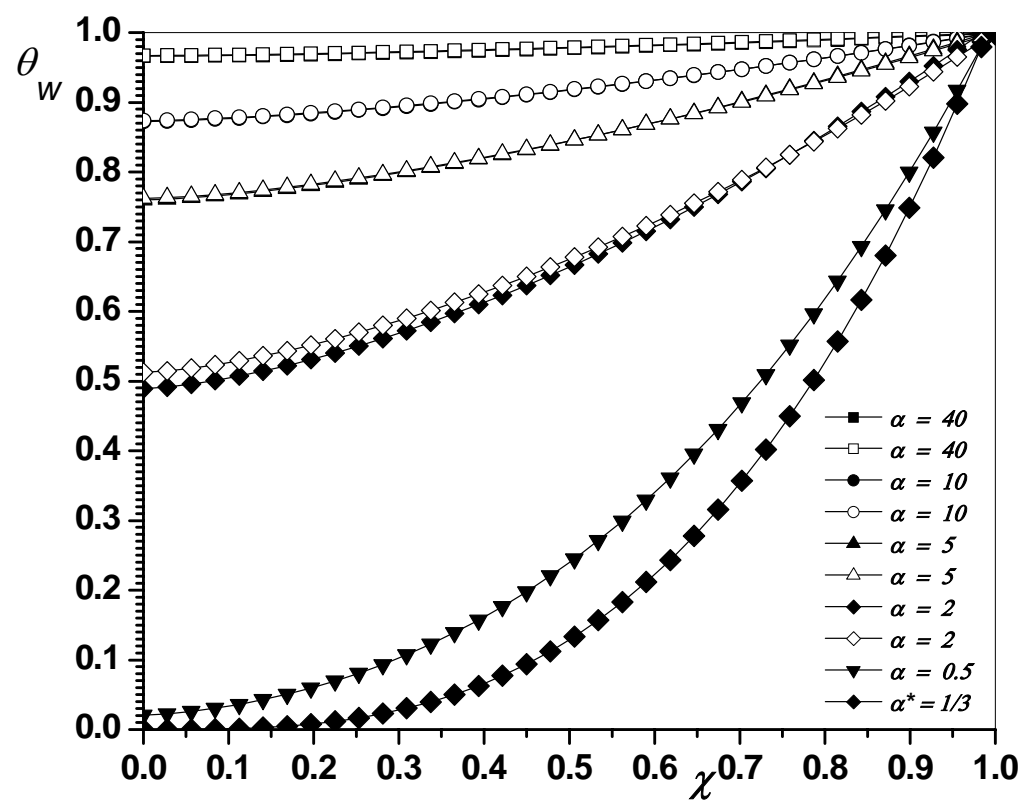

Figure 3: Nondimensional temperature of the fin, $\theta_{w}$, as a function of the nondimensional longitudinal coordinate $\chi$, for different values of the conjugate parameter $\alpha$ (black symbols denotes numerical solutions and 
white symbols are analytical solutions).

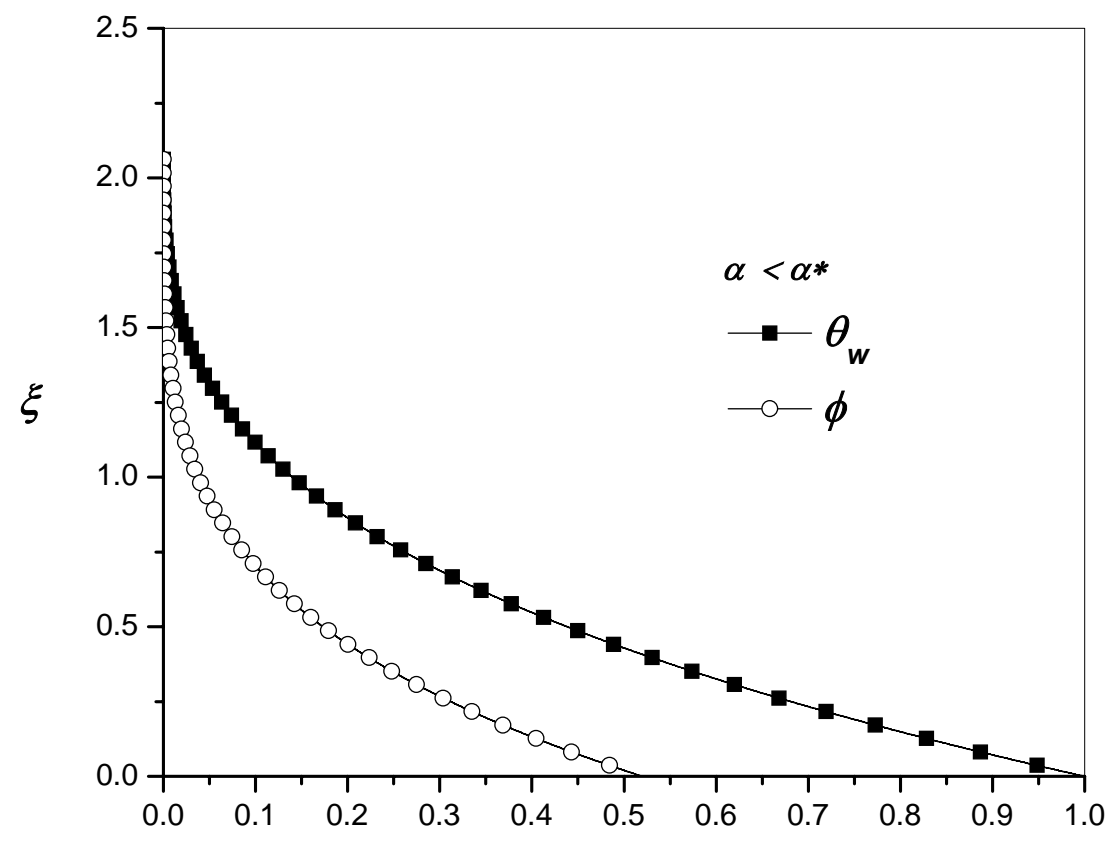

Figure 4: Nondimensional temperature, $\theta_{w}$ (- symbol) and modified thickness of the condensed film, $\phi$ ( $\circ$ symbol) as a function of the nondimensional inner coordinate $\xi$ for $\alpha<\alpha^{*}$.

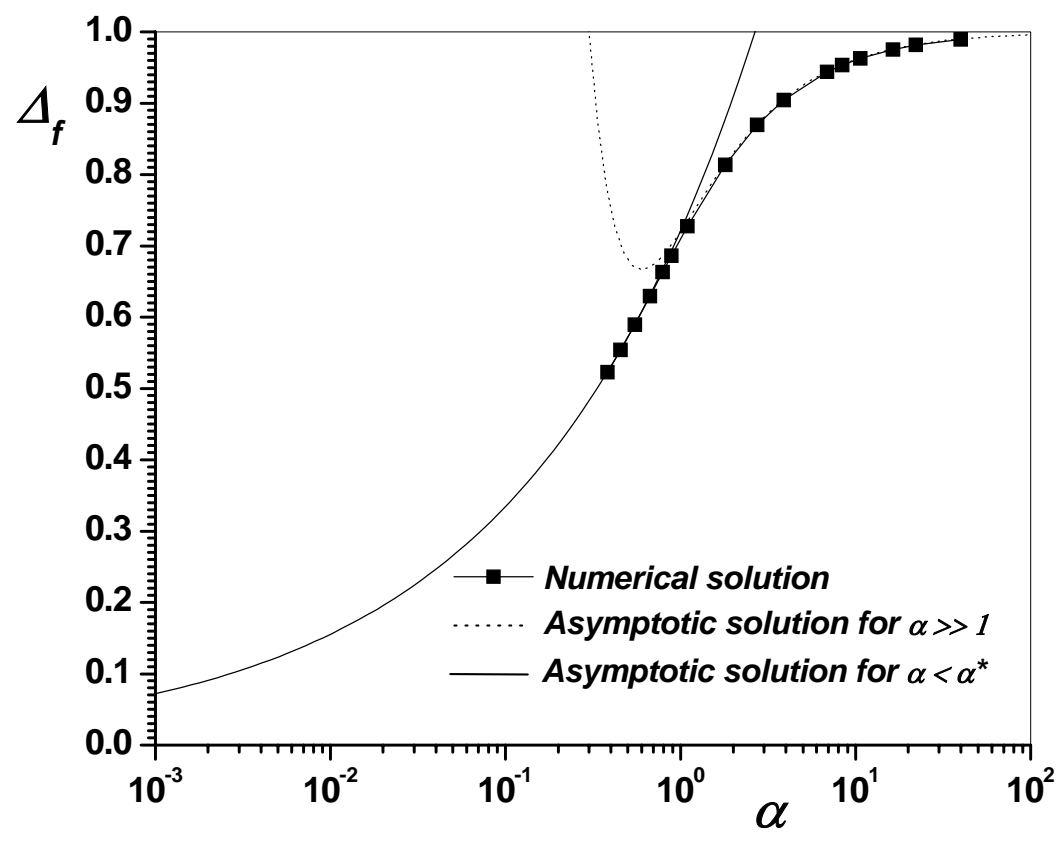


Figure 5: Nondimensional thickness of the condensed film at the base of the fin, $\Delta_{f}$, as a function of the conjugate parameter $\alpha$. The asymptotic solutions for $\alpha>>1$ and $\alpha<\alpha^{*}$ are also plotted.

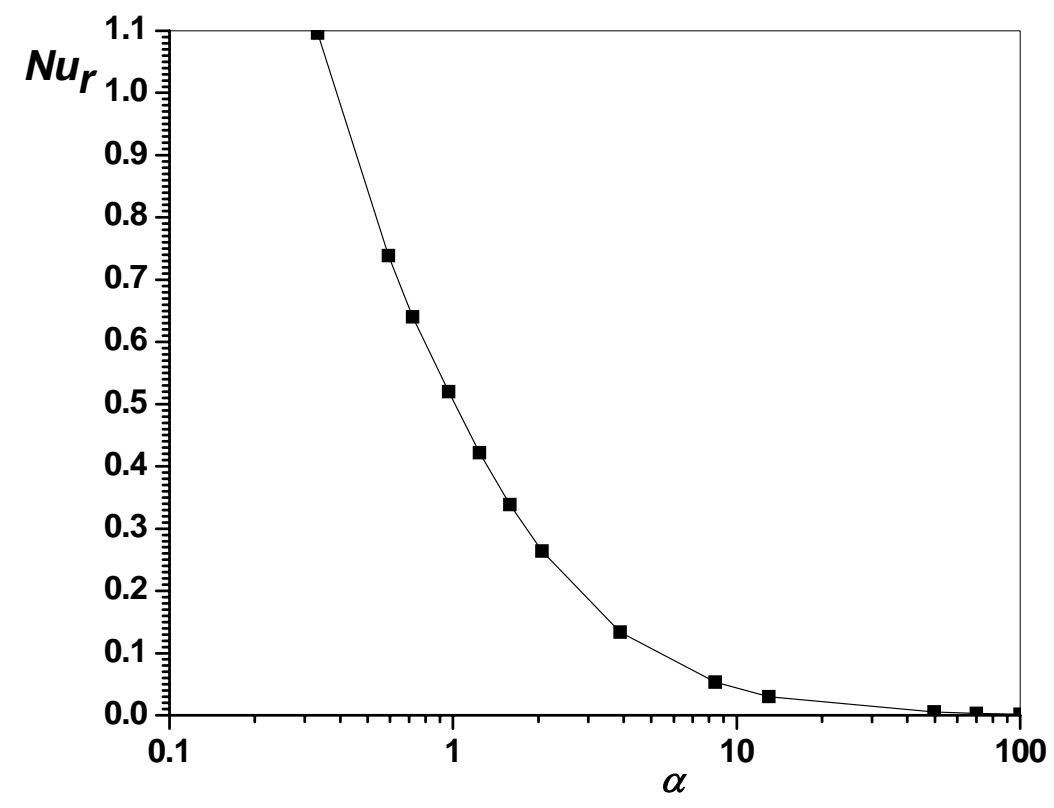

Figure 6: Average reduced Nusselt number as a function of the conjugate parameter $\alpha$.

\section{REFERENCES}

Bejan A. Convection Heat Transfer, pp. 146-150, John Wiley \& Sons; 1984.

Chen HT, Lan Z, Wang TI. Study of conjugate conduction-laminar film condensation for a vertical plate fin. Int. J. Heat Mass Transfer, 37: 2592-2597, 1994.

Chen MM. An analytical study of laminar film condensation, Part 1 flat plates. J. Heat Transfer, 83: 48-54, 1961.

Chen SL, Gerner FM, Tien CL. General film condensation correlations. Experimental Heat Transfer, 1: 93-107, 1987.

Cheng P. Film condensation along an inclined surface in a porous medium. Int. J. Heat Mass Transfer, 24: 983-990, 1981.

Incropera F. P., DeWitt D. P. Fundamentals of Heat and Mass Transfer. John Wiley \& Sons; 1996.

Kaviany M, Principles of Heat Transfer in Porous Media. Springer-Verlag, 1995.

Kibboua R, Azzi A, Laminar film condensation on an elliptical tube embedded in porous media, Energy Conversion and Management, 46: 2359-2372, 2005.

Koh JCY, Sparrow EM, Hartnett JP. The two phase boundary layer in laminar film condensation. Int. J. Heat Mass Transfer, 2: 69-82, 1961.

Koh JCY. On integral treatment of two-phase boundary layer in film condensation. J. Heat Transfer, 83: 359-362, 1961.

Méndez F, Lizardi JJ, Treviño C, Laminar film condensation along a vertical fin. Int. J. Heat 
Mass Transfer, 43: 2859-2868, 2000.

Méndez F, Treviño C. Film condensation generated by a forced cooling fluid. Eur. J. Mech. B/Fluids, 15: 217-240, 1996.

Méndez F, Treviño C. Film condensation induced by a natural convective flow: steady-state analysis. Int. J. Heat Mass Transfer, 40: 1279-1289, 1997.

Merte Jr. H. Condensation heat transfer. Adv. Heat Transfer, 15: 181-272, 1973.

Nield DA, Bejan A. Convection in Porous Media, pp. 426-428, Springer-Verlag, 1998.

Patankar SV, Sparrow EM. Condensation on an extended surface. J. Heat Transfer, 101: 434440, 1979.

Sarma PK, Chary SP, Dharma Rao V. Condensation on a vertical plate fin of variable thickness. Int. J. Heat Mass Transfer, 31: 1941-1944, 1988.

Sparrow EM, Gregg JL. A boundary layer treatment of laminar-film condensation. Trans. Am. Soc. Mech. Engrs, 81: 13-17, 1959.

Treviño C, Becerra G, Méndez F. Laminar film condensation on a thin finite thickness plate. AIAA Journal of Thermophysics and Heat Transfer, 11: 119-121, 1997.

Wilkins JE. Condensation on an extended surface. J. Heat Transfer, 02: 186-187, 1980. 